\title{
Influence of spore morphology on spectrophotometric quantification of Trichoderma inocula
}

\author{
$\overline{\text { Georg Schuitz }^{1,2} \text {, Dietmar Haltrich }{ }^{1,2} \text { \& Lea Atanasova*,1 }}$
}

\begin{abstract}
Species of the genus Trichoderma are filamentous fungi commonly used in research, industry and agriculture. Trichoderma reesei strains are prominent producers of cellulolytic and hemicellulolytic enzymes as well as being expression hosts; several other species such as $T$. atroviride might be exploited as biocontrol agents. A careful preparation of Trichoderma inocula, which consists mainly of conidia (asexual spores), is of immense importance. Conidia concentration is still mostly determined with the help of a hemocytometer; however, as a more accurate and time-saving alternative, absorbance can be used to estimate fungal spore counts. We established a spectrophotometric method for fast and reliable preparation of Trichoderma inocula by evaluating the effect of size, shape and pigmentation of the conidia at different wavelengths.
\end{abstract}

\section{METHOD SUMMARY}

A fast and easy method was developed to quantify fungal spore concentrations in aqueous solution with emphasis on the size, shape and pigmentation of Trichoderma conidia.

\section{KEYWORDS}

conidia $\cdot$ hemocytometer $\cdot$ spectrophotometer $\cdot$ spore concentration - spore suspension - Trichoderma atroviride - Trichoderma reesei

${ }^{1}$ Department of Food Sciences \& Technology, BOKU-University of Natural Resources \& Life Sciences, Vienna, Austria; ${ }^{2}$ Doctoral Program BioToP, Biomolecular Technology of Proteins, BOKU-University of Natural Resources \& Life Sciences Vienna, Vienna, Austria; *Author for correspondence:lea.atanasova@boku.ac.at

BioTechniques 68: 279-282 (May 2020) 10.2144/btn-2019-0152
Members of the genus Trichoderma (Hypocreales, Ascomycota) are ubiquitous filamentous fungi with high biotechnological and agricultural relevance. Some species, like Trichoderma reesei, grow on dead wood and bark, and are well known for their production of cellulolytic and hemicellulolytic enzymes [1]. A strain of $T$. reesei, named QM6a and isolated from the cotton canvas of a US Army tent during World War II, became the reference strain during the following decades [2]. It was also the parental strain used in many academic and industrial strain improvement programs, mostly with the goal of increasing or decreasing cellulase production [3]. For instance, two rounds of irradiating conidia of QM6a in a linear particle accelerator resulted in the overproducing strain QM9414 with four-times higher cellulase levels than QM6a [4]. Later, deletion of the $x y r 1$ gene, involved in transcriptional regulation of the major xylanolytic and cellulolytic enzymes in $T$. reesei, resulted in a cellulase-negative mutant suitable for recombinant protein production $[5,6]$. Beside the efficient secretion of hydrolytic enzymes, T. reesei produces various secondary metabolites such as nonribosomal peptides and polyketides. Investigations of the polyketide synthase-encoding gene pks 4 showed that it is responsible for the green conidial pigmentation, and its deletion resulted in the production of hyaline conidia and white stroma [7].

Another species, $T$. atroviride, and here especially the fungicide-resistant strain P1 (ATCC 74058), is very well studied for its biocontrol abilities. This species is frequently isolated from soil and the rhizosphere of many plants, and is of immense importance in agriculture because of the beneficial effects on crops and other plants that result from its activity against many phytopathogenic fungi $[8,9]$.

Whether in industry, academia or agriculture, working with filamentous fungi requires preparation of inocula with precise and reproducible conidia concentrations, since variations greatly affect experimental outcomes. The use of hemocytometers to quantify conidia is still widespread; in addition to the limitations of accuracy for dilute and very concentrated spore suspensions, this method is also timeconsuming, especially when working with large numbers of strains or replicates. As an alternative, optical density or absorbance measurements have been in use for decades [10] to determine concentrations of bacterial and fungal suspensions. When using this approach, absorbance should be plotted against particle count to generate a calibration curve for estimation of the particle concentration for each species. Valdez and Piccolo [11] used optical density at $340 \mathrm{~nm}$ to quantify spores of Penicillium allii on necrotic garlic tissue, having to deal with interfering soluble garlic compounds. Inocula of Geotrichum candidum and Trichoderma viride were measured at $550 \mathrm{~nm}$; however, only for spore concentrations lower than $9 \times 10^{6} \mathrm{cells} / \mathrm{ml}[12,13]$. Morris and Nicholls [15] used optical density over a broader range of concentrations for G. candidum and two Penicillium spp. [14]. A cumbersome infrared reflectance spectrophotometry method (at 1900, 2252 and 2308 $\mathrm{nm}$ ) was established for powdery mildews and rusts. The influence of size and shape for bacteria and mitochondria [10], as well as for larger fungal conidia [14] has been investigated previously; however, the effect of multiple morphological characteristics of conidia on the calibration curve has so far not yet been considered.

Here we present a fast and accurate spectrophotometric method to measure fungal conidia concentrations over a wide spectral range in aqueous solutions. We tested this method with different strains of the two most commonly used Trichoderma species, $T$. reesei $(T r)$ and $T$. atroviride $(T a)$, which differ in conidial morphology. The influence of conidial size, shape and pigmentation on the absorbance of fungal inocula at various wavelengths 


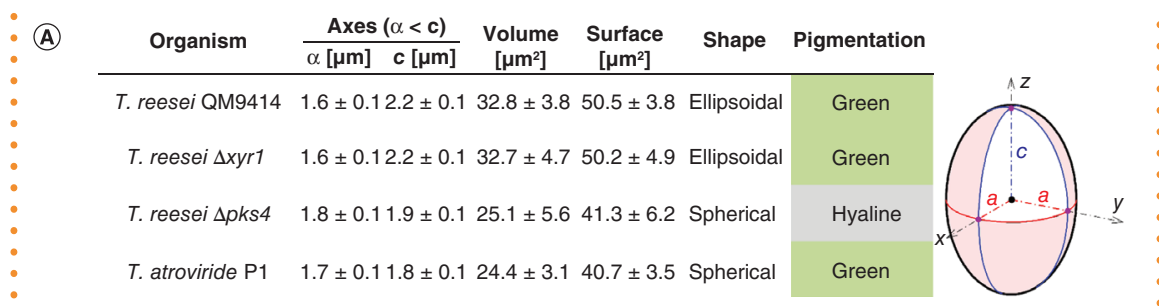

(B)

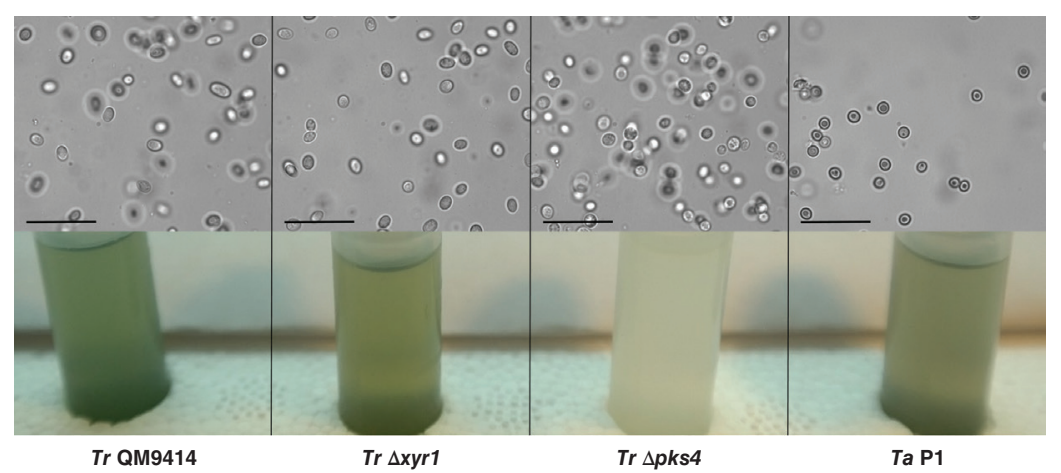

Figure 1. (A) Size, volume, surface, shape and conidial pigmentation of Trichoderma strains used in this study. Size and shape were measured under $100 \times$ magnification with the automated inverted bright-field microscope (Leica DMI6000 B) in $0.05 \%$ Tween $^{\circledast} 20$. The average of $n=30$ conidia (semiaxes: $\alpha<c$ ) was used to calculate the volume and surface area using the equations for a prolate spheroid. (B) Conidial morphology (size and shape) and conidial pigmentation based on the color of spore suspensions from selected $T$. reesei and $T$. atroviride strains. Scale bars represent $20 \mu \mathrm{m}$. Ta: Trichoderma atroviride; Tr: Trichoderma reesei.

was evaluated. We used Tr QM9414 [4] as a commonly used parental strain for many genetically modified $\operatorname{Tr}$ strains, the QM9414 $\Delta x y r 1$ deletion mutant $[5,6]$ to determine the influence of putative variations in conidia as a consequence of gene

manipulation, the $\operatorname{Tr} \Delta p k s 4$ mutant [7] due to its translucid conidia, and Ta P1 [8] because of its smaller conidial size compared with Tr QM9414.

Spore solutions of the strains were homogeneously spread out on $2.4 \%$ potato

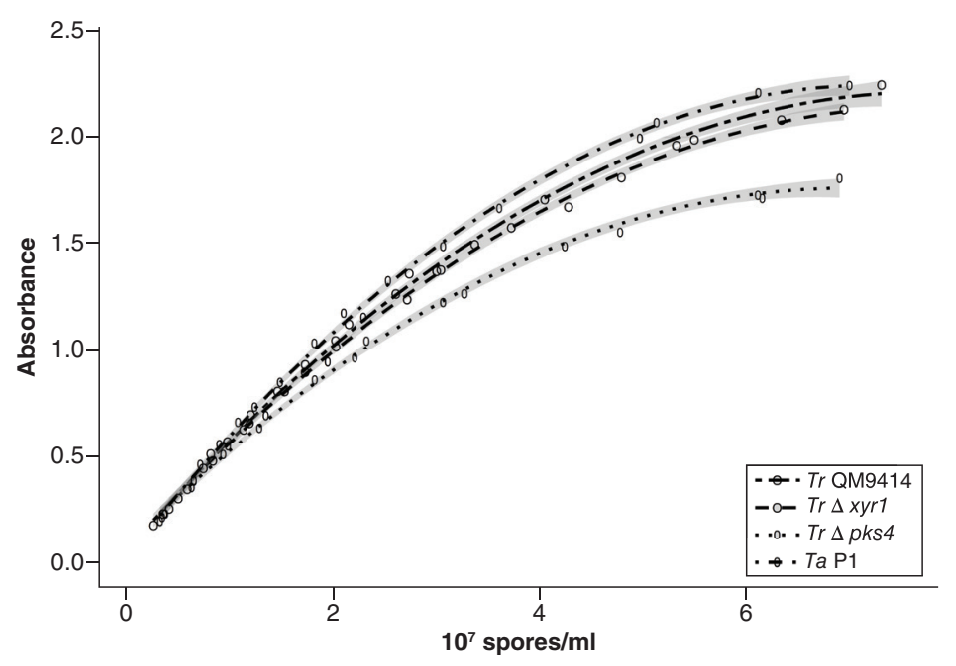

Figure 2. Calibration curves of the combined biological replicates of all four investigated Trichoderma spp. for the whole dataset measured at $700 \mathrm{~nm}$ (datasets for 550 and $600 \mathrm{~nm}$ showed equivalent results). Curves of green-pigmented conidia ( $\operatorname{Tr}$ QM9414, $\operatorname{Tr} \Delta x y r 1$ and $T a$ P1) group close together. The gray area surrounding the curves represents the coefficient intervals.

Ta: Trichoderma atroviride; Tr: Trichoderma reesei. dextrose agar (BD Difco, Heidelberg, Germany) plates and cultivated at $28^{\circ} \mathrm{C}$ for $>7$ days until the entire plates were covered with conidia (Supplementary Figure 1). Conidia of the same age were harvested by spreading $5 \mathrm{ml}$ of a $0.05 \%$ Tween $^{\circledR} 20$ solution (Sigma-Aldrich, MO, USA) on the plates and lifting the cells gently into suspension. Suspensions were then vortexed vigorously for $1 \mathrm{~min}$ and filtered through sterile glass wool. Freshly prepared Trichoderma inocula were used to determine the conidial morphology of all strains. $\operatorname{Tr}$ QM9414 and Tr $\Delta x y r 1$ conidia were more ellipsoidal (ANOVA, $p<0.05$ ) and slightly larger compared to Tr $\triangle p k s 4$ and Ta P1 (ANOVA, $\mathrm{p}<0.05$ ) (Figure 1A \& B).

To generate the calibration curve from each conidial suspension, a dilution series was prepared with $0.05 \%$ Tween ${ }^{\circledR} 20$ solution as diluent. Absorbance was determined for all conidial suspensions at 550,600 and 700 nm using a Hitachi U-3000 spectrophotometer, and conidia were counted using a Thoma hemocytometer (Carl Roth, Karlsruhe, Germany) following the manufacturer's protocol. Each biological replicate was tested three times for absorbance and hemocytometer measurements. The average of the technical replicates was used to plot absorbance versus the number of conidia per milliliter, resulting in eight independent calibration curves (two biological replicates for each of the four Trichoderma strains). The calibration curves were in good correlation with the general form of a second-degree polynomial function:

Abs $=10^{7}$ spores $/ \mathrm{ml}^{*} b_{2}^{2}+10^{7}$ spores $/ \mathrm{ml}^{*} b_{1}+b_{0}$ with $b_{0}, b_{1}$ and $b_{2}$ as coefficients for any $b_{0^{\prime}} b_{1} \in \mathbb{R} ; b_{2} \in \mathbb{R} \backslash\{0\}$. The coefficients of both curves were compared (R version 3.5.2 and RStudio Version 1.1.463) for each biological replicate pair and showed no significant difference (ANOVA, $p>0.05$ ), thus they were combined to one curve for each tested strain (Figure 2).

To analyze the combined calibration curves of each fungus in terms of differences in size and pigmentation, we used the full range of data related by a second-degree polynomial function. In general, the curvature of standard curves is mainly caused by multiple scattering, and this effect is reduced for smaller particles [16]. Additionally, standard curves at longer wavelengths 

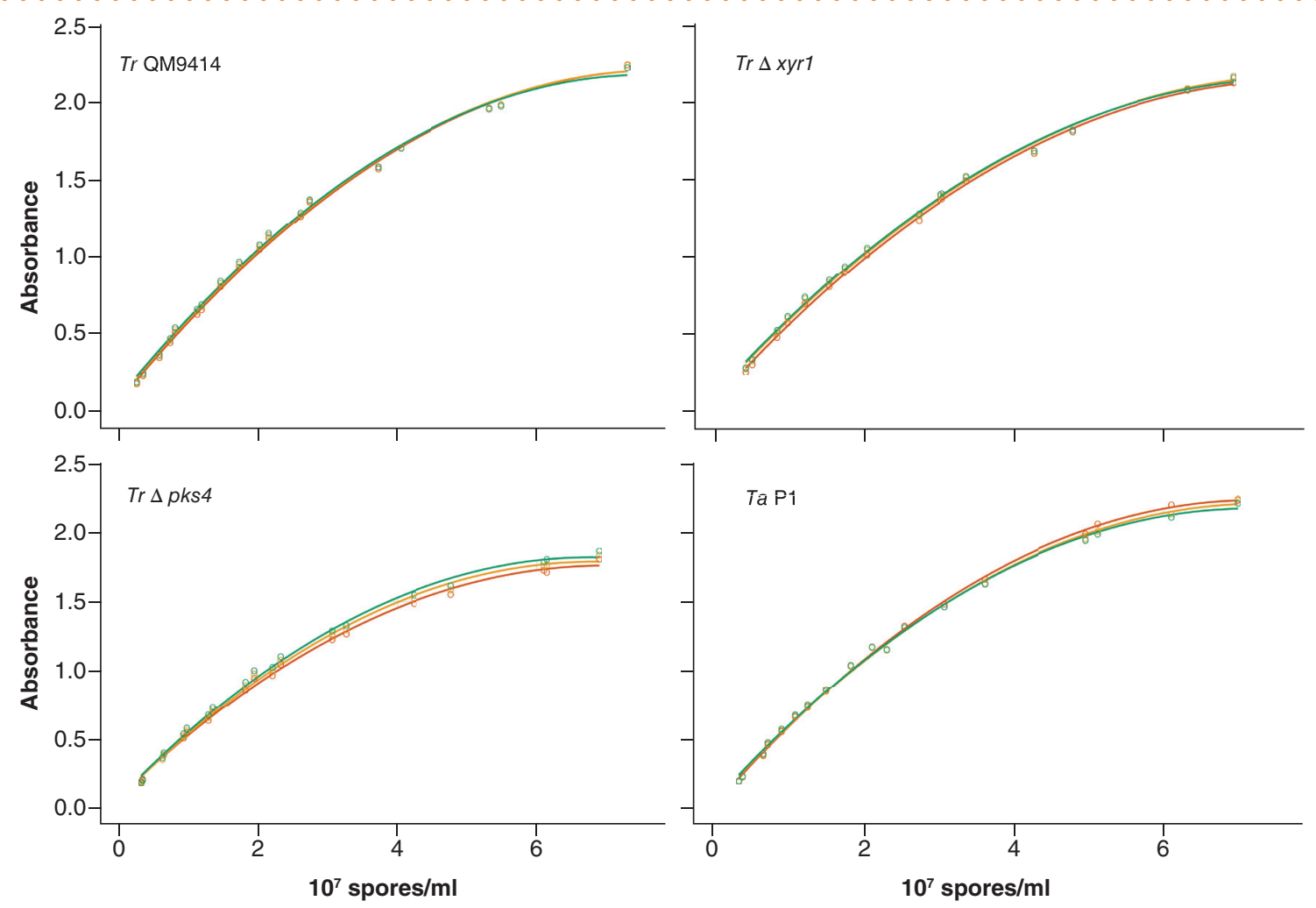

Figure 3. Relationship between spore concentration and absorbance compared at $550 \mathrm{~nm}$ (green), $600 \mathrm{~nm}$ (orange) and $700 \mathrm{~nm}$ (red) for $T$. reesei and T. atroviride strains. Calibration curves for each strain at 550,600 and $700 \mathrm{~nm}$ show no significant distinction (ANOVA, $p>0.05$ ). Ta: Trichoderma atroviride; Tr: Trichoderma reesei.

produce straighter curves, are affected less by the use of different spectrophotometers, and show a decreased amount of absorption due to particle pigmentation according to several studies $[14,16,17]$. Our results show that the effect of straighter curves at longer wavelengths can be disregarded above $550 \mathrm{~nm}$. This can be observed for all four Trichoderma strains when comparing the coefficients of the calibration curves at 550, 600 and $700 \mathrm{~nm}$, showing that there is no significant distinction (ANOVA, $p>0.05$ ) between the measurements at different wavelengths for each strain (Figure 3).

In spectrophotometric experiments, multiple scattering can lead to lower absorbance than expected, since more light is detected when photons are redirected back to the detector after an additional scattering event. This effect is more pronounced for dense suspensions [10]. The scattering cross-section $s_{S}$ is a measure of the shape and size of the particles at constant wavelength with the number of particles present extrapolated to zero. In this study, all four strains had very similar $s_{S}$ values of
$6.9 \times 10^{-8}, 6.7 \times 10^{-8}, 6.6 \times 10^{-8}$ and $6.9 \times 10^{-8}$ $\mathrm{cm}^{2}$ (ANOVA, $\mathrm{p}>0.05$ ), which were calculated as described in [17], for $\operatorname{Tr}$ QM9414, $\operatorname{Tr} \Delta x y r 1$, $\operatorname{Tr} \Delta p k s 4$ and $T a \mathrm{P} 1$, respectively (Supplementary Figure 2). Therefore the small

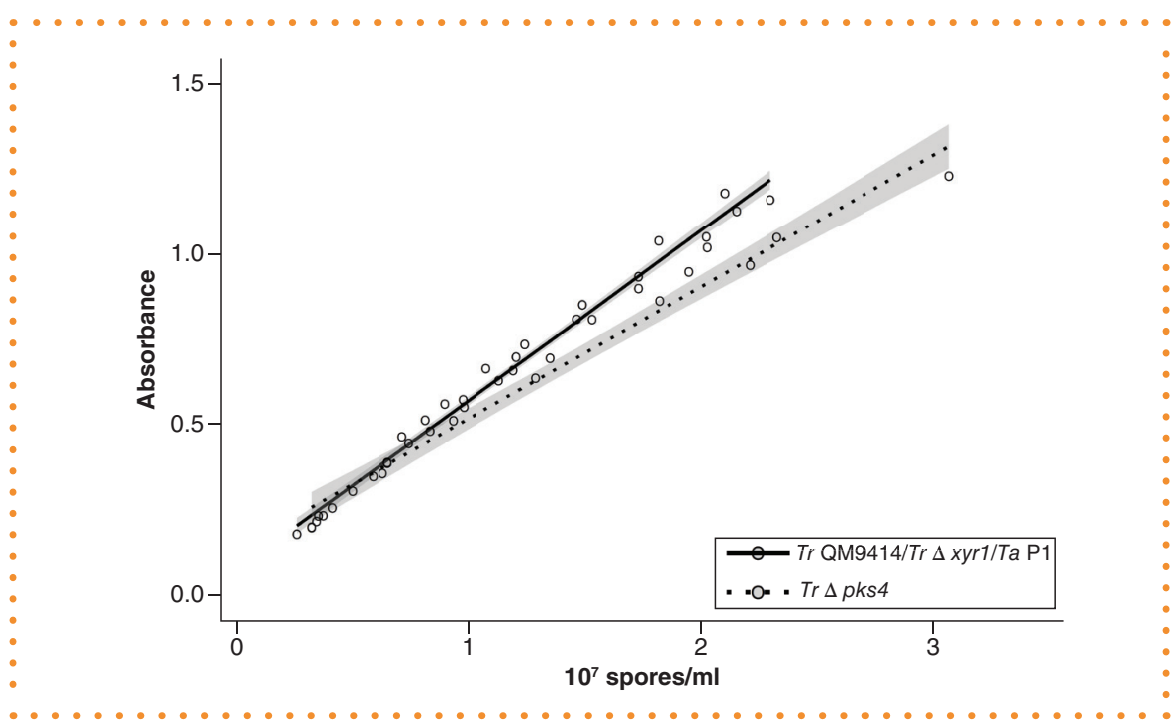

Figure 4. Relation between conidia concentration and absorbance $(<1.2)$ at $700 \mathrm{~nm}$ for $T$. reesei $\Delta p k s 4$ with hyaline conidia and values combined for green-pigmented conidia (T. reesei QM9414, T. reesei $\triangle x y r 1$ and $T$. atroviride $\mathrm{P} 1)$. The gray area surrounding the lines represents the confidence intervals.

Ta: Trichoderma atroviride; Tr: Trichoderma reesei. differences in size and shape, as observed between $\operatorname{Tr}$ QM9414 and $\operatorname{Tr} \Delta x y r 1$ compared with $\operatorname{Tr} \Delta p k s 4$ and Ta P1 (Figure 1A), can be ignored. Our results show that pigmentation was the most important factor affecting the 
Table 1. Linear regression coefficients and equations for estimating conidia count ( $\left.10^{7} \mathrm{spores} / \mathrm{ml}\right)$ from absorbance measurements at $700 \mathrm{~nm}$ (datasets for 550 and $600 \mathrm{~nm}$ showed equivalent results) for $T$. reesei $\triangle$ pks 4 with hyaline conidia, and separated and combined strains with green-pigmented conidia ( $T$. reesei QM9414, T. reesei $\Delta x y r 1$ and $T$. atroviride $\mathrm{P} 1)$.

Organism Calibration curves for $10^{7}$ spores $/ \mathrm{ml}$ $R^{2}$

T. reesei QM9414 $=2.015 \pm 0.083 * A b s-0.135 \pm 0.058$ 0.99

T. reesei $\triangle x y r 1$ $=2.098 \pm 0.143 * A b s-0.167 \pm 0.098$

0.99

T. reesei $\Delta p k s 4$ $=2.548 \pm 0.111 * A b s-0.309 \pm 0.078$ 0.98

T. atroviride $\mathrm{P} 1$ $=1.926 \pm 0.113 * A b s-0.125 \pm 0.082$ 0.98

Combined strains with green conidia

$=1.985 \pm 0.051$ * Abs $-0.128 \pm 0.037$

0.99

calibration curves of conidia. Calibration curves of strains with green conidia did not show any significant differences when measured at 550, 600 or $700 \mathrm{~nm}$ (ANOVA, $p>0.05)$. In contrast, the standard curve of the $\operatorname{Tr} \Delta p k s 4$ strain with hyaline conidia showed statistically lower absorbance at the same number of conidia compared with all strains with green conidia (ANOVA, $p<0.05$ ) (Figures 2 \& 3 ).

For fast and easy application in laboratory use, we chose the dataset measured at $700 \mathrm{~nm}$ and restricted absorbance values to a maximum of 1.2 because of the effect of curvature and the resulting loss of accuracy at higher absorbance values (Figure 4). Within this data range, absorbance is closely related to the number of conidia by a linear regression, and was fitted in the inverse way to yield easy-to-use equations in the general form:

$10^{7}$ spores $/ \mathrm{ml}=b_{1} * \mathrm{Abs}+b_{0}$

Moreover, the datasets of the strains with green conidia (Tr QM9414, Tr $\Delta x y r 1$ and Ta P1) could be combined to generate one calibration curve, whereas strain $\operatorname{Tr} \Delta p k s 4$ with hyaline conidia was significantly different at all three wavelengths used (ANOVA, $p<0.05$ ) (Figure 4 \& Table 1).

Summarizing our results, absorbance between 550 and $700 \mathrm{~nm}$ is directly related to conidial count for all four Trichoderma strains, and therefore the calibration curves can be used as a convenient and rapid tool to estimate conidia concentration in a solution. While no differences in the individual calibration curves could be detected when measuring at different wavelengths $(550,600$ and $700 \mathrm{~nm})$, pigmentation of conidia has a strong effect on the slope of a calibration curve, in contrast to the negligible differences in size and shape among different Trichoderma conidia.

\section{SUPPLEMENTARY DATA}

To view the supplementary data that accompany this paper please visit the journal website at: www.future-science. com/doi/suppl/10.2144/btn-2019-0152

\section{AUTHOR CONTRIBUTIONS}

G Schütz and D Haltrich conceived the study; $G$ Schütz and $L$ Atanasova designed the experiments; G Schütz, L Atanasova and D Haltrich wrote the manuscript; G Schütz performed the experiments, analyzed the data, and prepared the figures and supplementary files.

\section{ACKNOWLEDGMENTS}

The authors would like to thank M Melcher for guidance with $\mathrm{R}$ and solutions of statistical problems, M Debreczeny and the BOKU Imaging Center for help with the imaging equipment.

\section{FINANCIAL \& COMPETING INTERESTS DISCLOSURE}

This work was supported by Austrian Science Fund (FWF) grants W1224 (Doctoral program BioToP - Biomolecular Technology of Proteins) to D Haltrich and P 30460-B32 to $L$ Atanasova. Open access funding was provided by the FWF. The authors have no other relevant affiliations or financial involvement with any organization or entity with a financial interest in or financial conflict with the subject matter or materials discussed in the manuscript apart from those disclosed.

No writing assistance was utilized in the production of this manuscript.

\section{OPEN ACCESS}

This work is licensed under the Creative Commons Attribution 4.0 License. To view a copy of this license, visit http://creativecommons.org/licenses/by/4.0/

\section{REFERENCES}

1. Atanasova L. Ecophysiology of Trichoderma in genomic perspective. Biotechnol. Biol. Trichoderma 25-40 (2014).

2. Reese E. History of the cellulase program at the U.S. army Natick. Biotechnol. Bioeng. Symp. 6, 9-20 (1976).

3. Martinez D, Berka RM, Henrissat B et al. Genome sequencing and analysis of the biomass-degrading
fungus Trichoderma reesei (syn. Hypocrea jecorina). Nat. fungus Trichoderma reesei (syn. Hypoc
Biotechnol. 26(5), 553-560 (2008).

4. Mandels M, Weber J, Parizek R. Enhanced cellulase production by a mutant of Trichoderma viride. Appl. Microbiol. 21(1), 152-154 (1971).

5. Stricker AR, Grosstessner-Hain K, Würleitner E, Mach RL. Xyr1 (Xylanase Regulator 1) regulates both the hydrolytic enzyme system and D-xylose metabolism in Hypocrea jecorina. Eukaryot. Cell 5(12), 2128-2137 (2006).

6. Uzbas F, Sezerman U, Hartl L, Kubicek CP, Seiboth B. A homologous production system for Trichoderma reesei Microbiol. Biotechnol. 93(4), 1601-1608 (2012).

7. Atanasova L, Knox BP, Kubicek CP, Druzhinina IS, Baker $\mathrm{SE}$. The polyketide synthase gene pks 4 of Trichoderma reesei provides pigmentation and stress resistance. Eukaryot. Cell 12(11), 1499-1508 (2013).

8. Kubicek CP, Sukno S, Gruber S et al. Comparative genome sequence analysis underscores mycoparasitism as the ancestral life style of Trichoderma. Genome Biol. 12(4), R40 (2011).

9. Nordberg $\mathrm{H}$, Cantor M, Dusheyko $\mathrm{S}$ et al. The genome portal of the Department of Energy Joint Genome Institute: 2014 updates. Nucleic Acids Res. 42(D1), 26-31 (2014).

10. Koch AL. Some calculations on the turbidity of mitochondria and bacteria. Biochim. Biophys. Acta 51(3), 429-441 (1961).

11. Valdez JG, Piccolo RJ. Use of spectrophotometry as a tool to quantify the sporulation of Penicillium allii in garlic lesions. Fitopatol. Bras. 31(6), 595-597 (2006).

12. Wells J, Spalding D. Stimulation of Geotrichum candidum by low oxygen and high carbon dioxide atmospheres. Phytopathology 65, 199-1302 (1975).

13. Waghunde RR, John P, Naik BM, Solanky KU, Sabalpara AN. Optical density - a tool for the estimation of spore count of Trichoderma viride. J. Biopesticides 3(3), 624-626 (2010).

14. Morris SC, Nicholls PJ. An evaluation of optical density to estimate fungal spore concentrations in water suspension. Phytopathology 68(8), 1240-1242 (1978).

15. Asher MJC, Cowe IA, Thomas CE, Cuthbertson DC. A rapid method of counting spores of fungal pathogens by infrared reflectance analysis. Plant Pathol. 31, 363-371 (1982).

16. Koch AL. Turbidity measurements of bacterial cultures in some available commercial instruments. Anal. Biochem. 38(1), 252-259 (1970).

17. Koga S, Fujita T. Total cross section for optical scattering by spherical cells in suspension. J. Gen. Appl. Microbiol. 6(2), 101-107 (1960). 